\title{
AMBIENT QUASICONFORMAL HOMOGENEITY OF PLANAR DOMAINS
}

\author{
Petra Bonfert-Taylor, Richard D. Canary, Gaven Martin, \\ Edward C. Taylor and Michael Wolf \\ Wesleyan University, Department of Mathematics \\ 265 Church Street, Middletown, CT 06459, U.S.A.; pbonfert@wesleyan.edu \\ University of Michigan, Department of Mathematics
}

2074 East Hall, 530 Church Street, Ann Arbor, MI 48109-1043, U.S.A.; canary@umich.edu

Massey University, Institute of Information and Mathematical Sciences

Albany, Auckland, New Zealand; g.j.martin@massey.ac.nz

Wesleyan University, Department of Mathematics

265 Church Street, Middletown, CT 06459, U.S.A.; ectaylor@wesleyan.edu

Rice University, Department of Mathematics

P.O. Box 1892, Houston, TX 77251, U.S.A.; mwolf@math.rice.edu

\begin{abstract}
We prove that the ambient quasiconformal homogeneity constant of a hyperbolic planar domain which is not simply connected is uniformly bounded away from 1 .

We also consider a component $\Omega_{0}$ of the domain of discontinuity of a finitely generated Kleinian group $\Gamma$. We show that if $\Omega_{0} / \Gamma$ is compact, then $\Omega_{0}$ is uniformly ambiently quasiconformally homogeneous, and that if $\Omega_{0}$ is not simply connected and its quotient $\Omega_{0} / \Gamma$ is non-compact, then $\Omega_{0}$ is not uniformly quasiconformally homogeneous.
\end{abstract}

\section{Introduction}

An orientable hyperbolic manifold $N$ is called $K$-quasiconformally homogeneous if for any $x, y \in N$, there exists a $K$-quasiconformal automorphism of $N$ taking $x$ to $y$. In earlier work [6], the authors established that for any $n \geq 3$ there exists $K_{n}>1$ such that if $N$ is a $K$-quasiconformally homogeneous hyperbolic $n$-manifold, other than $\mathbf{H}^{n}$, then $K \geq K_{n}$. It is natural to ask whether or not such a constant can be found in dimension 2 (see, for example, [5]).

For planar domains, one can define a more restrictive notion of quasiconformal homogeneity. An open set $\Omega \subseteq \widehat{\mathbf{C}}$ is ambiently $K$-quasiconformally homogeneous if, for all $x, y \in \Omega$, there exists a $K$-quasiconformal homeomorphism $f: \widehat{\mathbf{C}} \rightarrow \widehat{\mathbf{C}}$ such that $f(x)=y$ and $f(\Omega)=\Omega$. We will say that a planar domain $\Omega$ is uniformly ambiently quasiconformally homogeneous if there exists some $K$ such that $\Omega$ is ambiently $K$-quasiconformally homogeneous. Sarvas [21] showed that any ambiently $K$-quasiconformally homogeneous Jordan domain is a quasidisk. As any Jordan domain is conformally homogeneous, we see that, in general, ambient quasiconformal homogeneity is much stronger than quasiconformal homogeneity.

doi:10.5186/aasfm.2010.3516

2000 Mathematics Subject Classification: Primary 30C62; Secondary 30F45.

Key words: Quasiconformal homogeneity, hyperbolic planar domain, Kleinian groups, quasiconformal removability.

Bonfert-Taylor and Taylor were partially supported by the National Science Foundation grant DMS-0706754, Canary was partially supported by NSF grants DMS-0504791 and DMS-0554239, Martin was partially supported by the Marsden Fund of New Zealand, and Wolf was partially supported by NSF grant DMS-0505603. 
Our first main result shows that the ambient quasiconformal homogeneity constant is uniformly bounded away from 1 for hyperbolic planar domains which are not simply connected. Notice that any $K$-quasidisk (which is not round) is ambiently $K$ quasiconformally homogeneous, but not ambiently 1-quasiconformally homogeneous (see Gehring-Palka [12] and Erkama [11]) so one cannot bound the constant away from 1 in the simply connected case.

Theorem 1.1. There exists $K_{0}>1$ such that if $\Omega$ is an ambiently K-quasiconformally homogeneous hyperbolic planar domain which is not simply connected, then $K \geq K_{0}$.

It is clear that for a planar domain whose complement is removable for $K$ quasiconformal maps, $K$-quasiconformal homogeneity is equivalent to ambient $K$ quasiconformal homogeneity. Recall that a closed subset $R$ of $\widehat{\mathbf{C}}$ is removable for L-quasiconformal maps if whenever $f: \widehat{\mathbf{C}}-R \rightarrow \widehat{\mathbf{C}}$ is $L$-quasiconformal, then it admits a $L$-quasiconformal extension to a map $\bar{f}: \widehat{\mathbf{C}} \rightarrow \widehat{\mathbf{C}}$. Thus, we obtain the following corollary:

Corollary 1.2. If $\Omega$ is a hyperbolic, $K$-quasiconformally homogeneous planar domain which is not simply connected, and $\widehat{\mathbf{C}}-\Omega$ is removable for $L$-quasiconformal maps, then $K \geq \min \left\{K_{0}, L\right\}$.

Iwaniec and Martin [15, Theorem 11.3] showed that given any $d<1$, there exists $L_{d}>1$ such that any subset of $\widehat{\mathbf{C}}$ of Hausdorff dimension at most $d$ is removable for $L_{d}$-quasiconformal maps (see also the discussion in the Historical remarks at the end of the section). Thus, we obtain a lower bound on the usual uniform quasiconformal homogeneity constant for planar domains whose complements have small Hausdorff dimension. (Recall that any closed subset of $\widehat{\mathbf{C}}$ of Hausdorff dimension less than one is totally disconnected.)

Corollary 1.3. Given any $d<1$, there exists $K_{d}>1$ such that if $\Omega$ is a hyperbolic $K$-quasiconformally homogeneous planar domain whose complement has Hausdorff dimension at most $d$, then $K \geq K_{d}$.

A result of Martio, Rickman and Väisälä [19] shows that sets of zero capacity are quasiconformally removable (i.e., removable for $K$-quasiconformal maps for all $K)$. Moreover, Heinonen and Koskela [13] show that spherically porous sets are quasiconformally removable. So, we obtain:

Corollary 1.4. If $\Omega$ is a hyperbolic, $K$-quasiconformally homogeneous planar domain and either $\widehat{\mathbf{C}}-\Omega$ has zero capacity or is spherically porous, then $K \geq K_{0}$.

In a final section, we study quasiconformal homogeneity for components of domains of discontinuity of Kleinian groups. We recall that a Kleinian group $\Gamma$ is a discrete subgroup of $\mathrm{PSL}_{2}(\mathbf{C})$, regarded as the group of conformal automorphisms of $\widehat{\mathbf{C}}$, and that its domain of discontinuity $\Omega(\Gamma)$ is the largest open subset of $\widehat{\mathbf{C}}$ on which $\Gamma$ acts properly discontinuously. A Kleinian group $\Gamma$ is said to be analytically finite if $\Omega(\Gamma) / \Gamma$ is of finite type. Recall that Ahlfors [1] proved that every finitely generated Kleinian group is analytically finite and that there exist examples of infinitely generated Kleinian groups that are analytically finite.

We show that the quasiconformal homogeneity of a non-simply connected component $\Omega_{0}$ of the domain of discontinuity of a finitely generated Kleinian group is 
determined entirely by the compactness, or lack thereof, of the quotient Riemann surface $\Omega_{0} / \Gamma$. (In section 4 we will establish a quantitative version of this result.)

Theorem 1.5. Suppose that $\Omega_{0}$ is a component of the domain of discontinuity of a non-elementary analytically finite Kleinian group $\Gamma$.

(1) If $\Omega_{0} / \Gamma$ is compact, then $\Omega_{0}$ is uniformly ambiently quasiconformally homogeneous, and

(2) if $\Omega_{0} / \Gamma$ is non-compact and $\Omega_{0}$ is not simply connected, then $\Omega_{0}$ is not uniformly quasiconformally homogeneous.

If the limit set of the Kleinian group has Hausdorff dimension less than 1, then Corollary 1.3 allows one to obtain lower bounds on the quasiconformal homogeneity constant of the domain of discontinuity. It follows immediately from work of Canary and Taylor [10] that if the limit set of a finitely generated Kleinian group $\Gamma$ has Hausdorff dimension less than one and $\Omega(\Gamma) / \Gamma$ is compact, then $\Gamma$ has a finite index subgroup $\Gamma_{0}$ which is a Schottky group (i.e., $\mathbf{H}^{3} \cup \Omega\left(\Gamma_{0}\right) / \Gamma_{0}$ is homeomorphic to a handlebody.) The domain of discontinuity of a finitely generated Schottky group is known as a Schottky domain.

Corollary 1.6. If $\Omega$ is a $K$-quasiconformally homogeneous Schottky domain whose complement has Hausdorff dimension at most $d<1$, then $K \geq K_{d}$ where $K_{d}$ is the constant in Corollary 1.3.

We will use Theorem 1.5, see Example 4.1, to exhibit a uniformly ambiently quasiconformally homogeneous domain whose complement has infinitely many components, Hausdorff dimension 2 and measure zero.

Historical remarks. Quasiconformally homogeneous domains were first studied by Gehring and Palka [12]. Ambient quasiconformal homogeneity, and the stronger notion of quasiconformal bihomogeneity, were first introduced by MacManus, Näkki and Palka [17], where it is simply called quasiconformal homogeneity. (For further results on ambient quasiconformal homogeneity and bihomogeneity, see the paper by Bonfert-Taylor and Taylor [7].)

Gehring and Palka, see Lemma 4.3 in Gehring-Palka [12], showed that if the quotient of a component $\Omega_{0}$ of the domain of discontinuity of a Kleinian group is compact, then $\Omega_{0}$ is uniformly quasiconformally homogeneous. In fact, the argument they give also proves part (1) of Theorem 1.5 and we will essentially follow their argument. Example 4.1 is inspired by Example 4.6 in [12].

Astala, Clop, Mateu, Orobitg and Uriarte-Tuero [2] have sharpened the result of Iwaniec-Martin [15] to show that any set of $\sigma$-finite $\frac{2}{K+1}$-dimensionsal Hausdorff measure is removable for $K$-quasiconformal mappings. See section 13.5 of AstalaIwaniec-Martin [3] for further discussion of this and related issues.

In both [15] and [19] the removability results are stated for bounded quasiregular maps. For quasiconformal maps $f: \Omega \rightarrow \widehat{\mathbf{C}}$, we may normalize so that $\infty \in \Omega$ and $f(\infty)=\infty$, so, by considering $\left.f\right|_{\Omega-C}$ where $C \subset \Omega$ is a closed neighborhood of $\infty$, we are able to dispense with the boundedness assumptions in our statements.

Acknowledgements. The authors would like to acknowledge the support of the Banff International Research Station. The bulk of the research in this paper was carried out as part of a Focussed Research Group at Banff in March 2007.

The authors would also like to thank the referee for useful comments on the original version of this paper. 


\section{Basic facts}

In this section, we develop the background material necessary to establish our two main results, Theorems 1.1 and 1.5.

2.1. The ambient quasiconformal homogeneity constant. It is natural to define the ambient quasiconformal homogeneity constant of a uniformly ambiently quasiconformally homogeneous domain to be

$$
K_{\mathrm{amb}}(\Omega)=\inf \{K>1 \mid \Omega \text { is ambiently } K \text {-quasiconformally homogeneous }\} \text {. }
$$

A normal family argument (see Lemma 2.1 in [6]) shows that this infimum is achieved.

Lemma 2.1. If $\Omega$ is a uniformly ambiently quasiconformally homogeneous planar domain, then $\Omega$ is ambiently $K_{\mathrm{amb}}(\Omega)$-quasiconformally homogeneous.

Similarly, we recall that if a hyperbolic manifold $N$ is uniformly quasiconformally homogeneous, then we can define

$$
K(N)=\min \{K>1 \mid N \text { is } K \text {-quasiconformally homogeneous }\} .
$$

2.2. Bounded geometry. A key observation in the study of $K$-quasiconformally homogeneous hyperbolic manifolds is that they have bounded geometry. If $N$ is a hyperbolic $n$-manifold, then let $l(N)=2 \inf _{x \in N} \operatorname{inj}_{N}(x)$ and let $d(N)=$ $\sup _{x \in N} \operatorname{inj}_{N}(x)$, where $\operatorname{inj}_{N}(x)$ denotes the injectivity radius of $N$ at the point $x$.

Theorem 2.2. (Theorem 1.1 in [6]) For all $n$ and $K>1$, there exists $m(n, K)>$ 0 such that if $N$ is a $K$-quasiconformally homogeneous hyperbolic $n$-manifold other than $\mathbf{H}^{n}$, then

(1) $d(N) \leq K l(N)+2 K \log 4$, and

(2) $l(N) \geq m(n, K)$.

This result has a few immediate corollaries for quasiconformally homogeneous planar domains. Recall that a compact set $A$ in $\widehat{\mathbf{C}}$ is uniformly perfect if there exists $K$ such that all annuli in $\widehat{\mathbf{C}} \backslash A$ that separate $A$ have modulus at most $K$. For example, Pommerenke [20] showed that the limit set of a finitely generated, nonelementary Kleinian group is uniformly perfect. Canary [8] observed that the limit set of an analytically finite Kleinian group is uniformly perfect (see also [16]).

Corollary 2.3. If $\Omega \subset \widehat{\mathbf{C}}$ is a uniformly quasiconformally homogeneous hyperbolic planar domain, and $\Lambda=\widehat{\mathbf{C}}-\Omega$, then

(1) $\Lambda$ is uniformly perfect,

(2) $\Lambda$ does not have isolated points, and

(3) if $\Omega$ is not simply connected, then $\Omega$ has infinitely generated fundamental group.

Proof. By Theorem 2.2, there is a positive lower bound on the injectivity radius in $\Omega$. The existence of such a bound is equivalent to uniform perfectness of $\Omega$ 's complement, by Theorem 1 in [20]. This establishes (1).

If $\Lambda$ has an isolated point, then $\Omega$ would contain annuli with arbitrarily large moduli that separate $\Lambda$. This contradiction establishes (2).

Recall that any complete non-compact surface having finitely generated nontrivial fundamental group, does not have bounded geometry, i.e. either it has points with arbitrarily large injectivity radius or points with injectivity radius arbitrarily 
close to 0 . Therefore, since $\Omega$ is non-compact and has bounded geometry, it must have infinitely generated fundamental group if it is not simply connected, which establishes (3).

2.3. Ambient conformal homogeneity. If an open set is ambiently 1quasiconformally homogeneous, we say that it is ambiently conformally homogeneous. One may combine Theorem 8.1 of Gehring-Palka [12] with the main result of Erkama [11] to obtain a complete characterization of ambiently conformally homogeneous domains.

Proposition 2.4. An open set $\Omega$ in $\widehat{\mathbf{C}}$ is ambiently conformally homogeneous if and only if $\Omega=\widehat{\mathbf{C}}, \Omega$ is a round disk, $\Omega$ is the complement of a round circle in $\widehat{\mathbf{C}}$ or $\Omega$ is the complement of one or two points in $\widehat{\mathbf{C}}$.

2.4. Carathéodory convergence. We recall that a sequence $\left\{\Omega_{n}\right\}$ of open sets in $\widehat{\mathbf{C}}$ converges to an open set $\Omega \subset \widehat{\mathbf{C}}$ in the sense of Carathéodory if the following are satisfied:

(1) If $C \subset \Omega$ is compact, then there exists $N$ such that $C \subset \Omega_{n}$ if $n \geq N$, and

(2) if an open set $U$ is contained in $\Omega_{n}$ for infinitely many values of $n$, then $U \subset \Omega$.

This type of convergence is also known as kernel convergence. Notice that we allow the limit $\Omega$ to be empty.

We recall that every sequence of planar domains has a subsequence which converges in the sense of Carathéodory and that $\left\{\Omega_{n}\right\}$ converges to $\Omega$ in the sense of Carathéodory if and only if $\Lambda=\widehat{\mathbf{C}}-\Omega$ is the Hausdorff limit of the complements $\left\{\Lambda_{n}=\widehat{\mathbf{C}}-\Omega_{n}\right\}$.

\section{A lower bound on the ambient quasiconformal homogeneity constant}

In this section we give the proof of Theorem 1.1. We recall the statement of the theorem for the reader's convenience.

Theorem 1.1. There exists $K_{0}>1$ such that if $\Omega$ is a uniformly ambiently quasiconformally homogeneous hyperbolic planar domain which is not simply connected, then

$$
K_{\mathrm{amb}}(\Omega) \geq K_{0}
$$

We proceed by contradiction. We assume that there exists a sequence $\left\{\Omega_{n}\right\}$ of ambiently $K_{n}$-quasiconformally homogeneous planar domains which are not simply connected such that $\lim K_{n}=1$. Then we normalize appropriately and study the Carathéodory limit of a convergent subsequence to obtain a contradiction. In particular, we note that the proof does not yield an explicit estimate for $K_{0}$.

We may assume that $\infty \in \partial \Omega_{n}$ for all $n$, where $\partial \Omega_{n}=\overline{\Omega_{n}}-\Omega_{n} \subset \widehat{\mathbf{C}}$. Let $\alpha_{n}$ be a homotopically non-trivial simple closed curve in $\Omega_{n}$. Let $\Lambda_{n}=\widehat{\mathbf{C}}-\Omega_{n}$ and let $A_{n}$ be the portion of $\Lambda_{n}$ inside the region enclosed by $\alpha_{n}$ and let $B_{n}$ be the portion of $\Lambda_{n}-\{\infty\}$ lying outside the region enclosed by $\alpha_{n}$. Since $\infty$ is not an isolated point of $\Lambda_{n}$ (by Corollary 2.3), $B_{n}$ is non-empty. Let $a_{n} \in A_{n}$ and $b_{n} \in B_{n}$ be points which minimize the (Euclidean) distance between $A_{n}$ and $B_{n}$. We may assume, by normalizing by a similarity of $\mathbf{C}$, that $a_{n}=-1$ and $b_{n}=1$. By construction $A_{n}$ cannot intersect the open ball of radius 2 about 1 , while $B_{n}$ cannot intersect the 
open ball of radius 2 about -1 . Therefore, the intersection $D$ of the open ball of radius 2 about 1 and the open ball of radius 2 about -1 must be contained in $\Omega_{n}$.

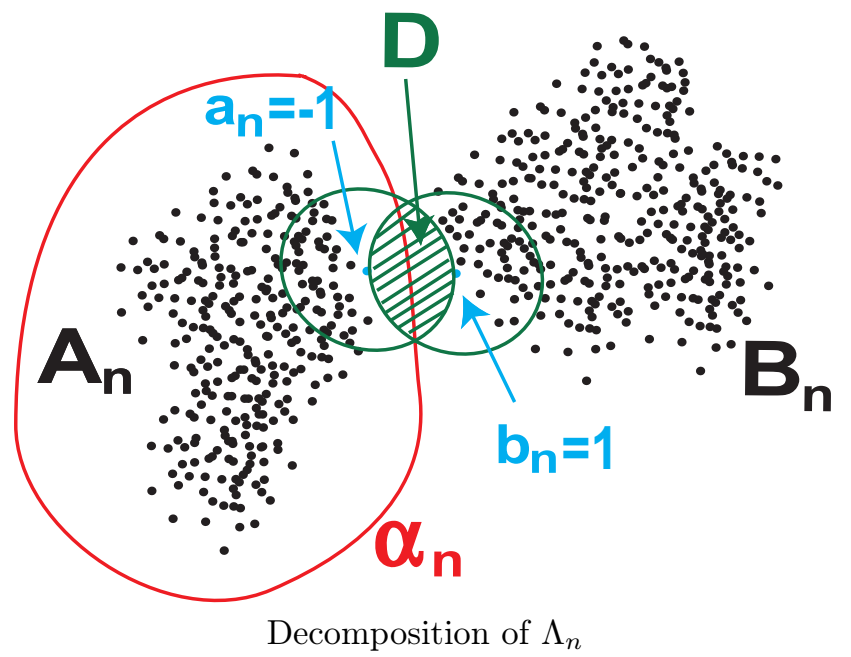

We now pass to a subsequence so that $\left\{\Omega_{n}\right\}$ converges, in the sense of Carathéodory, to a planar domain $\Omega$. Since $D$ is open and contained in $\Omega_{n}$ for all $n$, we see that $D \subset \Omega$. Since, $1,-1$ and $\infty$ do not lie in $\Omega_{n}$ for any $n$, they must not lie in $\Omega$ either. In particular, $-1,1 \in \partial \Omega$.

We now claim that $\Omega$ is ambiently conformally homogeneous. Let $x, y \in \Omega$, then $x, y \in \Omega_{n}$ for all large enough $n$ (by the definition of Carathéodory convergence). So there exists, for all large $n$, a $K_{n}$-quasiconformal map $f_{n}: \widehat{\mathbf{C}} \rightarrow \widehat{\mathbf{C}}$ such that $f_{n}(x)=y$ and $f_{n}\left(\Omega_{n}\right)=\Omega_{n}$. We can pass to a subsequence such that either

(1) $\lim f_{n}=f$ and $f$ is conformal, or

(2) $f_{n}$ converges, uniformly on compact subsets of $\widehat{\mathbf{C}}-\left\{x_{0}\right\}$, for some point $x_{0} \in \widehat{\mathbf{C}}$, to a constant map with image $y$

(see, for example, Corollaries 21.3 and 37.2 in Väisälä [24]).

In case $(2)$, either $x_{0} \neq 1$ or $x_{0} \neq-1$. Assuming that $x_{0} \neq 1$, we see that $\lim f_{n}(1)=y$ which would imply that $y \in \Lambda=\widehat{\mathbf{C}}-\Omega$ since $f_{n}(1) \in \Lambda_{n}$ for all $n$ and $\Lambda$ is the Hausdorff limit of $\left\{\Lambda_{n}\right\}$. If $x_{0}=1$, then $\lim f_{n}(-1)=y$ and again we conclude that $y \in \Lambda$. This is a contradiction, so we must be in case (1).

In case (1) it remains to show that $f(\Omega)=\Omega$. If $z \in \Lambda$, then there exists $z_{n} \in \Lambda_{n}$ such that $\lim z_{n}=z$ (since $\Lambda$ is the Hausdorff limit of $\Lambda_{n}$ ). So, since $f_{n}\left(\Lambda_{n}\right)=\Lambda_{n}$ for all $n, f_{n}\left(z_{n}\right) \in \Lambda_{n}$ and, again since $\Lambda$ is the Hausdorff limit of $\Lambda_{n}$, we see that $f(z)=\lim f_{n}\left(z_{n}\right) \in \Lambda$. Therefore, $f(\Lambda) \subset \Lambda$. But, we may similarly show that $f^{-1}(\Lambda) \subset \Lambda$. Since $f$ is a homeomorphism, this implies that $f(\Omega)=\Omega$ as desired. Therefore, there exists a conformal map $f: \widehat{\mathbf{C}} \rightarrow \widehat{\mathbf{C}}$ such that $f(x)=y$ and $f(\Omega)=\Omega$. Since $x$ and $y$ were arbitrary elements in $\Omega$, it follows that $\Omega$ is ambiently conformally homogeneous.

Since $\Omega$ is ambiently conformally homogeneous, it has one of the forms described in Proposition 2.4. Since $\widehat{\mathbf{C}}-\Omega$ contains at least three points, it follows that $\Omega$ must be a round disk or the complement of a round circle. However, in either case $\partial \Omega$ must be a circle passing through -1 and 1 . But, any circle passing through 1 and -1 must intersect $D$ which is contained in $\Omega$. This contradiction completes the proof. 


\section{Quasiconformal homogeneity and Kleinian groups}

In this section, we establish a quantitative version of Theorem 1.5 and use it to construct various examples of uniformly ambiently quasiconformally homogeneous domains.

Theorem 1.5. (Quantitative version) Suppose that $\Omega_{0}$ is a component of the domain of discontinuity of an analytically finite Kleinian group $\Gamma$.

(1) If $\Omega_{0} / \Gamma$ is compact, let $D$ denote the diameter of $\Omega_{0} / \Gamma$ and let $L=\frac{l\left(\Omega_{0}\right)}{4}=$ $\frac{1}{2} \inf _{z \in \Omega_{0}} \operatorname{inj}_{\Omega_{0}}(z)$. Then $\Omega_{0}$ is ambiently $K$-quasiconformally homogeneous where

$$
K=\left(e^{L}+1\right)^{\frac{4 D+2 L}{L}},
$$

if $\Omega_{0}$ is not simply connected, and

$$
K=\left(e^{D}+1\right)^{2},
$$

if $\Omega_{0}$ is simply connected.

(2) If $\Omega_{0} / \Gamma$ is non-compact and $\Omega_{0}$ is not simply connected, then $\Omega_{0}$ is not uniformly quasiconformally homogeneous.

Proof of Theorem 1.5. Let $\Omega_{0}$ be a component of the domain of discontinuity of an analytically finite Kleinian group $\Gamma$.

We first suppose that $\Omega_{0} / \Gamma$ is compact. Notice that since $\Lambda_{\Gamma}$ is uniformly perfect, there is a positive lower bound for $\operatorname{inj}_{\Omega_{0}}(z)$, so $L>0$. There exists a compact convex fundamental domain $F$ for the action of $\Gamma$ on $\Omega_{0}$ of diameter at most $2 D$ (in the Poincaré metric on $\Omega_{0}$.) Let $U$ be a neighborhood of $F$ in $\Omega_{0}$ of radius $2 L$. The argument in Lemma 2.6 in [6], which is itself an application of Lemma 3.2 of Gehring-Palka [12], then implies that if $x, y \in F$, then there exists a $K$ quasiconformal automorphism $f: \widehat{\mathbf{C}} \rightarrow \widehat{\mathbf{C}}$ such that $f(x)=y$ and $f$ is the identity on $\widehat{\mathbf{C}}-U$ where

$$
K=\left(e^{L}+1\right)^{2\left(\frac{2 D}{L}+1\right)} .
$$

To be more precise, there exists a sequence of points $x=x_{0}, \ldots, x_{n}=y$ in $F$ such that $d\left(x_{i-1}, x_{i}\right)<L$ and $n \leq \frac{2 D}{L}+1$. Lemma 2.5 in [6] assures us that for all $i$ we can construct a $\left(e^{L}+1\right)^{2}$-quasiconformal map $f_{i}: \widehat{\mathbf{C}} \rightarrow \widehat{\mathbf{C}}$ which is the identity off the ball of radius $2 L$ about $x_{i}$ and $f_{i}\left(x_{i-1}\right)=x_{i}$. The map $f$ can then be taken to be $f_{n} \circ \cdots \circ f_{1}$. In the case that $\Omega_{0}$ is simply connected one may apply Lemma 2.5 from [6] directly to construct a $\left(e^{D}+1\right)^{2}$-quasiconformal map $f: \widehat{\mathbf{C}} \rightarrow \widehat{\mathbf{C}}$ which takes $x$ to $y$ and is the identity off of the ball of radius $2 D$ about $x$ in $\Omega_{0}$.

Now suppose that $z, w \in \Omega_{0}$. Then there exist elements $\alpha, \beta \in \Gamma$ such that $\alpha(z) \in F$ and $\beta(w) \in F$. By the argument above there exists a $K$-quasiconformal automorphism $f: \widehat{\mathbf{C}} \rightarrow \widehat{\mathbf{C}}$ such that $f(\alpha(z))=\beta(w)$ and $f\left(\Omega_{0}\right)=\Omega_{0}$. Then $g=$ $\beta^{-1} \circ f \circ \alpha$ is a $K$-quasiconformal automorphism of $\widehat{\mathbf{C}}$ such that $g\left(\Omega_{0}\right)=\Omega_{0}$ and $g(z)=w$. It follows that $\Omega_{0}$ is ambiently $K$-quasiconformally homogenous. We have established (1).

If $\Omega_{0} / \Gamma$ is not compact, then, since it has finite type, it contains a subsurface $C$ which is a canonical neighborhood of a cusp. To be more explicit, $C$ is homeomorphic to $S^{1} \times(0, \infty)$ and the metric is given by $c d \theta^{2}+e^{-2 t} d t^{2}$ for some $c>0$. Let $\tilde{C}$ be a component of the pre-image of $C$ in $\Omega_{0}$. If the covering of $C$ by $\tilde{C}$ is finite-to-one, 
then the injectivity radius of $\Omega_{0}$ (in its associated Poincaré metric) achieves values arbitrarily close to 0 within $\tilde{C}$. (We note that this case cannot actually occur when $\Gamma$ is analytically finite, since $\Omega(\Gamma)$ is known to be uniformly perfect.) Otherwise, $\tilde{C}$ is isometric to the universal cover of $C$, which is a horodisk, and the injectivity radius of $\Omega$ obtains values arbitrarily close to $\infty$ in $\tilde{C}$. Since the injectivity radius of a uniformly quasiconformally homogeneous surface, which is not simply connected, is bounded between two positive constants (see Theorem 2.2), it follows that $\Omega_{0}$ is not uniformly quasiconformally homogeneous, which establishes (2). This completes the proof of Theorem 1.5.

Theorem 1.5 provides many examples of ambiently quasiconformally homogeneous domains which are not even homeomorphic to conformally homogeneous domains. Schottky domains are one class of examples, but the following example indicates that the geometric behavior of these domains can be much worse.

Example 4.1. There exists a uniformly ambiently quasiconformally homogeneous domain $\Omega$ such that $\widehat{\mathbf{C}}-\Omega$ has infinitely many components, Hausdorff dimension 2, measure zero, and is not homeomorphic to a Cantor set.

Construction of Example 4.1. We recall that a finitely generated, geometrically infinite Kleinian group $\Gamma$ is said to be a degenerate group if its domain $\Omega(\Gamma)$ of discontinuity is connected and simply connected (and $\Gamma$ does not contain an abelian subgroup of finite index.) Let $\Gamma_{1}$ and $\Gamma_{2}$ be two degenerate groups such that $\Omega\left(\Gamma_{1}\right) / \Gamma_{1}$ and $\Omega\left(\Gamma_{2}\right) / \Gamma_{2}$ are both compact. For each $i$, let $F_{i}$ be a compact, convex fundamental domain for the action of $\Gamma_{i}$ on $\Omega\left(\Gamma_{i}\right)$. We may conjugate $\Gamma_{2}$ so that the closure of $\widehat{\mathbf{C}}-F_{1}$ is contained in the interior of $F_{2}$, the closure of $\widehat{\mathbf{C}}-F_{2}$ is contained in the interior of $F_{1}$, and the interior of $F_{1} \cap F_{2}$ contains a simple closed curve $W$ which separates $\Lambda_{\Gamma_{1}}$ from $\Lambda_{\Gamma_{2}}$.

If we let $\Gamma$ be the group generated by $\Gamma_{1}$ and $\Gamma_{2}$, then the Klein Combination Theorem (see Theorem VII.A.13 or Theorem VII.C.2 in Maskit [18]) implies that $\Gamma$ is a Kleinian group, $\Omega(\Gamma)$ is connected and $F_{1} \cap F_{2}$ is a fundamental domain for the action $\Gamma$ on $\Omega(\Gamma)$ and $\widehat{\mathbf{C}}-\Omega(\Gamma)$ has infinitely many components. Since $\Lambda\left(\Gamma_{1}\right) \subset \Lambda(\Gamma)$ is not totally disconnected, we see that $\Lambda(\Gamma)$ is not totally disconnected and hence is not homeomorphic to a Cantor set. Moreover, $\Omega(\Gamma) / \Gamma$ is compact and $\Gamma$ is finitely generated, so Theorem 1.5 implies that $\Omega(\Gamma)$ is uniformly ambiently quasiconformally homogeneous

It follows from Theorem 1 of Soma [22] that $\Gamma$ is geometrically tame, so one can apply work of Thurston [23] and Canary [9] to show that $\Lambda(\Gamma)=\widehat{\mathbf{C}}-\Omega(\Gamma)$ has measure zero. It is a consequence of work of Bishop and Jones [4] that $\Lambda(\Gamma)$ has Hausdorff dimension 2. Therefore, $\Omega=\Omega(\Gamma)$ has all the claimed properties.

Remark. Hjelle [14] exhibited simply connected ambiently quasiconformally homogeneous domains which are not quasidisks. We note that domains of discontinuity $\Omega(\Gamma)$ of degenerate Kleinian groups (such that $\Omega / \Gamma$ is compact) provide many such examples.

\section{References}

[1] Ahlfors, L.: Finitely generated Kleinian groups. - Amer. J. Math. 86, 1964, 413-429. 
[2] Astala, K., A. Clop, J. Mateu, J. Orobitg, and I. Uriarte-Tuero: Distortion of Hausdorff measures and improved Painlevé removability for quasiconformal mappings. - Duke Math. J. 141, 2008, 539-571.

[3] Astala, K., T. Inaniec, and G. Martin: Elliptic partial differential equations and quasiconformal mappings in the plane. - Princeton University Press, 2009.

[4] Bishop, C., and P. Jones: Hausdorff dimension and Kleinian groups. - Acta Math. 179, 1997, $1-39$.

[5] Bonfert-Taylor, P., M. Bridgeman, R. D. Canary, and E. C. Taylor: Quasiconformal homogeneity of hyperbolic surfaces with fixed-point full automorphisms. - Math. Proc. Cambridge Philos. Soc. 143, 2007, 71-84.

[6] Bonfert-Taylor, P., R. D. Canary, G. Martin, and E. C. Taylor: Quasiconformal homogeneity of hyperbolic manifolds. - Math. Ann. 331, 2005, 281-295.

[7] Bonfert-Taylor, P., and E. C. TAYlor: Quasiconformally homogeneous planar domains. Conform. Geom. Dyn. 12, 2008, 174-178.

[8] Canary, R. D.: The Poincaré metric and a conformal version of a theorem of Thurston. Duke Math. J. 64, 1991, 349-359.

[9] Canary, R. D.: Ends of hyperbolic 3-manifolds. - J. Amer. Math. Soc. 6, 1993, 1-35.

[10] Canary, R. D., and E. C. Taylor: Kleinian groups with small limit sets. - Duke Math. J. 73, 1994, 371-381.

[11] Erkama, T.: Möbius automorphisms of plane domains. - Ann. Acad. Sci. Fenn. Ser. A I Math. $10,1985,155-162$.

[12] Gehring, F. W., and B. PALKa: Quasiconformally homogeneous domains. - J. Anal. Math. 30, 1976, 172-199.

[13] Heinonen, J., and P. Koskela: Definitions of quasiconformality. - Invent. Math. 120, 1995, $61-79$.

[14] HJelle, G.: A simply connected, homogeneous domain which is not a quasidisk. - Ann. Acad. Sci. Fenn. Math. 30, 2005, 135-142.

[15] Imaniec, T., and G. Martin: Quasiregular mappings in even dimensions. - Acta Math. 170, 1993, 29-81.

[16] JÄRVI, P., and M. VUORINEN: Uniformly perfect sets and quasiregular mappings. - J. London Math. Soc. 54, 1996, 515-529.

[17] Macmanus, P., R. NÄKki, and B. Palka: Quasiconformally bi-homogeneous compacta in the complex plane. - Proc. London Math. Soc. 78, 1999, 215-240.

[18] Maskit, B.: Kleinian groups. - Springer-Verlag, 1988.

[19] Martio, O., S. RICKMan, and J. VÄIsÄLÄ: Distortion and singularities of quasiregular mappings. - Ann. Acad. Sci. Fenn. Ser. A I Math. 465, 1970, 1-13.

[20] Pommerenke, Ch.: On uniformly perfect sets and Fuchsian groups. - Analysis 4, 1984, 299321.

[21] Sarvas, J.: Boundary of a homogeneous Jordan domain. - Ann. Acad. Sci. Fenn. Ser. A I Math. 10, 1985, 511-514.

[22] SomA, T.: Function groups in Kleinian groups. - Math. Ann. 292, 1992, 181-190.

[23] Thurston, W. P.: The geometry and topology of 3-manifolds. - Lecture notes.

[24] VÄIsÄLÄ, J.: Lectures on n-dimensional quasiconformal mappings. - Springer-Verlag, 1971. 